\title{
Prognostic significance of HSF2BP in lung adenocarcinoma
}

\author{
Zhendong Huang ${ }^{1 *}$, Zhendong Liu ${ }^{2 \#}$, Xingbo Cheng ${ }^{2 \#}$, Zhibin Han ${ }^{2}$, Jiwei Li ${ }^{1}$, Tian Xia ${ }^{1}$, Yanzheng Gao ${ }^{2}$, \\ Li Wei ${ }^{1}$
}

${ }^{1}$ Department of Thoracic Surgery, Zhengzhou Key Laboratory for Surgical Treatment for End-Stage Lung Disease, Zhengzhou University People's Hospital, Henan Provincial People's Hospital, Zhengzhou, China; ${ }^{2}$ Department of Surgery of Spine and Spinal Cord, Henan Provincial People's Hospital, Henan International Joint Laboratory of Intelligentized Orthopedics Innovation and Transformation, Henan Key Laboratory for Intelligent Precision Orthopedics, Zhengzhou University People's Hospital, People's Hospital of Henan University, Zhengzhou, China

Contributions: (I) Conception and design: L Wei, Y Gao, Z Huang, Z Liu, X Cheng; (II) Administrative support: L Wei, Y Gao; (III) Provision of study materials or patients: J Li, T Xia; (IV) Collection and assembly of data: Z Huang, Z Liu, X Cheng, Z Han; (V) Data analysis and interpretation: Z Huang, Z Liu, X Cheng; (VI) Manuscript writing: All authors; (VII) Final approval of manuscript: All authors.

"These authors contributed equally to this work.

Correspondence to: Li Wei. Department of Thoracic Surgery, Zhengzhou University People's Hospital, Henan Provincial People’s Hospital, Henan University People's Hospital, No. 7 Weiwu Road, Jinshui District, Zhengzhou 450003, China. Email: wlixxt@sina.com; Yanzheng Gao. Department Of Orthopaedics, Zhengzhou University People's Hospital, Henan Provincial People's Hospital, No. 7 Weiwu Road, Jinshui District, Zhengzhou 450003, China. Email: doctorgao63@163.com.

Background: Recent studies have demonstrated that upregulation of heat shock transcription factor 2 binding protein (HSF2BP) may promote genomic instability, thereby leading to the development of tumors and also providing a potential target for biological antitumor therapy. However, the role of HSF2BP has so far remained unclear in lung adenocarcinoma (LUAD).

Methods: To explore the function of HSF2BP in LUAD, we collected transcriptome data for 551 lung samples from The Cancer Genome Atlas (TCGA) database and methylation data for 461 lung samples from the University of California Santa Cruz (UCSC) genome database, in addition to corresponding clinical information. We used bioinformatic approaches to systematically explore the role of HSF2BP in LUAD, including Gene Set Enrichment Analysis (GSEA), coexpression analysis, the Tumor IMmune Estimation Resource (TIMER) tool, Connectivity Map (CMap) analysis, and a meta-analysis involving three Gene Expression Omnibus (GEO) datasets and one TCGA dataset.

Results: Our results found that upregulation of HSF2BP in LUAD was an independent risk factor for the prognosis and diagnosis of LUAD. GSEA analysis showed HSF2BP expression was associated with vital signaling pathways, including the cell cycle, P53 signaling pathway, and homologous recombination. Coexpression analysis revealed $10 \mathrm{HSF} 2 \mathrm{BP}$-associated genes, including oncogenes and tumor suppressor genes. Additionally, we found that HSF2BP expression was negatively correlated with B-cell infiltration and had a potential interaction with CD80 in LUAD, which may play an important role in tumor immune escape. Finally, we identified four small-molecule drugs which show promise for LUAD treatment.

Conclusions: The present study found that elevated HSF2BP posed a threat to prognosis in LUAD patients. HSF2BP might have been involved in tumorigenesis by influencing genomic stability and contributing to tumor immune evasion in the tumor immune microenvironment of LUAD. These findings suggest that HSF2BP may provide a vulnerable target for improving and enhancing treatment of LUAD.

Keywords: Lung adenocarcinoma (LUAD); heat shock transcription factor 2 binding protein (HSF2BP); prognosis; immune response; small-molecule drugs

^ ORCID: 0000-0002-6091-6788. 
Submitted Aug 22, 2021. Accepted for publication Oct 13, 2021.

doi: 10.21037/atm-21-4935

View this article at: https://dx.doi.org/10.21037/atm-21-4935

\section{Introduction}

Lung adenocarcinoma (LUAD), with more than 1 million deaths each year worldwide, is the primary cause of cancerrelated death in lung cancer patients (1). With low-dose spiral computed tomography (CT) routinely employed in lung screening, an increasing number of patients with LUAD are diagnosed at an earlier stage and completely resected, improving the prognosis (2). In the meantime, the use of molecular-targeted therapies and immune checkpoint inhibitors, together with conventional chemotherapy and radiotherapy, has resulted in dramatic prognostic improvements in patients with advanced and metastatic LUAD (3-5). However, outcomes for LUAD patients generally continue to be dismal due to therapeutic resistance, tumor heterogeneity, and metastasis (6). Investigations of novel biomarkers for immunotherapy and optimizing combination therapy are imperative to provide a better prognosis for LUAD patients in the future (3).

Overexpression of heat shock proteins (HSPs, also known as molecular chaperones) has been found to be involved in proliferation, differentiation, invasion, metastasis, death, and immune recognition of tumor cells in a variety of human cancers $(7,8)$. Heat shock factors (HSFs), first identified by Italian geneticist Ferruccio Ritossa, are able to modulate the expression of HSPs and engage in tumorigenesis (9-12). In short, HSPs and HSFs, as the transcriptional activators of HSPs, are frequently cooperated in numerous biological processes. Upregulated expression of HSF2 has been found in a number of cancers, including esophageal squamous cell carcinoma, hepatocellular carcinoma, breast cancer, and lung cancer. HSF2 may lead to cell proliferation and migration by increasing the expression of HSPs in both nontumorigenic BEAS-2B cells and A549 lung cancer cells $(10,13)$. Heat shock transcription factor 2 binding protein (HSF2BP, also known as MEILB2) with 2 transcripts encoding a 334-aa polypeptide interacts with HSF2 and may be involved in modulating HSF2 activation (9). A recent study found that in human cancers, MEILB2-break repair meiotic recombinase recruitment factor 1 (BRME1) was often activated and impaired mitotic homologous recombination (14). Genomic instability caused by HSF2BP could promote tumor evolution and thus mutations that increase its expression would be positively selected in some tumors, including glioma and some breast, ovarian, and head and neck cancers but not in testicular germ cell tumors, which display reduced expression of HSF2BP $(15,16)$. In addition, Sato et al. demonstrated that high HSF2BP expression could suppress homologous recombination in DNA interstrand crosslink repair and increase cancer-promoting genomic instability, providing a targetable vulnerability (17).

Previous studies have demonstrated that elevated HSF2BP expression impaired genomic stability to induce tumorigenesis. However, the role of HSF2BP in the development of LUAD has not been investigated. In the present study, we systematically explored the role of HSF2BP in LUAD by analyzing RNA-sequencing (RNA-seq) data from 54 normal and 497 LUAD samples, together with clinical features from The Cancer Genome Atlas (TCGA) database. Our results were supported by a meta-analysis which included transcriptome data from three Gene Expression Omnibus (GEO) datasets and TCGA. In addition, epigenetic changes and immune infiltration characteristics containing clinical and molecular information, immune cells, and copy number alterations were also obtained. DNA methylation is crucial for the growth and development of various cells. Abnormalities of the DNA methylation level could alter transcriptome levels and deregulate cellular pathways. Our research found increasing methylation level of cg05527272 site could reduce HSF2BP expression. It is worth noting that this study examined the expression of HSF2BP in LUAD for the first time, thereby revealing its potential function in LUAD, particularly in relation to the immune microenvironment. We present the following article in accordance with the STARD reporting checklist (available at https://dx.doi. org/10.21037/atm-21-4935).

\section{Methods}

\section{Data mining}

We obtained transcriptome data of 551 specimens from TCGA (https://portal.gdc.cancer.gov/), including 54 normal and 497 LUAD tissue samples, to explore the differential expression of HSF2BP in tumor and nontumor samples. Clinical information and methylation changes derived from Xena Functional Genomics Explorer (https:// 
xenabrowser.net/) of 376 LUAD specimens were included to study the correlation between epigenetic changes, clinical traits, and HSF2BP expression. The study was conducted in accordance with the Declaration of Helsinki (as revised in 2013).

\section{Data processing of TCGA}

Of the 497 LUAD patients, 72 were excluded due to lack of survival status and/or clinical information. The remaining 425 cases were used to produce a Kaplan-Meier survival curve, and the log-rank test was used to test the significance of HSF2BP in prognosis of LUAD. Univariate and multivariate Cox proportional hazard models were used to calculate the hazard ratio (HR) of HSF2BP expression level and other clinical features in LUAD. HSF2BP was confirmed as an independent risk factor in prognosis of LUAD patients, elevated expression of which induced a worse overall survival (OS), so HSF2BP was preliminarily identified as a proto-oncogene.

\section{Gene Set Enrichment Analysis (GSEA) and coexpression analysis}

GSEA was utilized to indirectly assess the relevance between target genes and specific gene sets through the limma software package in $\mathrm{R}$ (3.6.2 version). The target gene was classified as either high or low expression based on the median HSF2BP expression level as the cut-off value. In addition, we conducted coexpression analysis using $\mathrm{R}$ software (version 3.6.2) to calculate the Pearson correlation coefficient for our target gene and other known genes, and then generated a circular graph to visualize the possible interactions between HSF2BP and other gene sets in the progress of LUAD.

\section{Tumor IMmune Estimation Resource (TIMER) analysis}

TIMER version 2.0 (https://cistrome.shinyapps.io/timer/), which provides clinical, immunological, and genomic features of various tumors, was used to determine the role of HSF2BP in the tumor immune microenvironment. A multivariable Cox proportional hazard model was employed to analyze clinical characteristics and HSF2BP expression in 455 LUAD patients. Kaplan-Meier plots were generated for the abundance of six immune infiltrates, including B cells, $\mathrm{CD}^{+} \mathrm{T}$ cells, $\mathrm{CD} 8^{+} \mathrm{T}$ cells, macrophages, neutrophils, and dendritic cells. We then investigated the correlation between the expression level of HSF2BP and the level of immune infiltration in LUAD and the potential interaction between HSF2BP and CD80. Finally, the relationship between somatic copy number alterations (SCNA) of HSF2BP in different immune cells and infiltration level in LUAD was analyzed.

\section{Analysis of Connectivity Map (CMap) database and Pubchem}

We used the CMap database of small-molecule drugs to identify potential therapies focusing on particular gene expression differences in order to theoretically develop a novel approach to improving treatment and prognosis of LUAD. On the basis of CMap data processing, we chose four drugs associated with LUAD. We then used the PubChem platform (https://pubchem.ncbi.nlm.nih.gov/) to locate the compound identifier (CID), molecular formula, and chemical structure for each drug.

\section{Meta-analysis}

In addition to the RNA-seq data obtained from TCGA, we used three datasets from platform GPL570 of the GEO (https://www.ncbi.nlm.nih.gov/geo/), including GSE31210, GSE37745, and GSE50081, comprising 226, 106, and 129 LUAD transcriptome samples, respectively. A meta-analysis was conducted using $\mathrm{R}$ software (version 3.6.2) to verify the association between the target gene and prognosis of LUAD. Due to low heterogeneity $\left(\mathrm{I}^{2}=32 \%<50 \% ; \mathrm{P}=0.22\right)$, we used a fixed-effects model.

\section{Statistical analysis}

The Cox regression model was implemented based on the "survival" package. "Limma" package was performed to conduct the normalization and differential analysis. Wilcoxon rank-sum test was mainly used for comparisons between two groups and Kruskal-Wallis test was utilized for two or more categories. All statistical analysis was conducted based on the $\mathrm{R}$ software (version 3.6.2). A twosided $\mathrm{P}$ value $<0.05$ was considered significant.

\section{Results}

\section{Associations between HSF2BP expression level, clinical features, and LUAD}

We processed RNA-seq data for 551 lung tissue specimens 


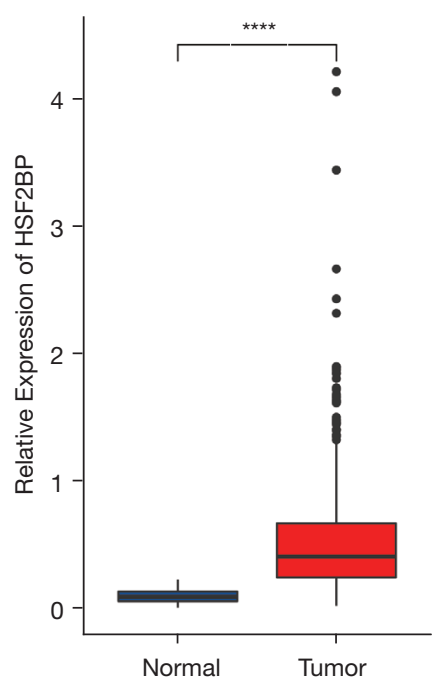

Figure 1 The expression of HSF2BP in normal $(\mathrm{N}=54)$ and LUAD ( $\mathrm{N}=497)$ tissues of human lung. ${ }^{* * * *}, \mathrm{P}<0.0001$. HSF2BP, heat shock transcription factor 2 binding protein; LUAD, lung adenocarcinoma.

from TCGA, including 54 normal control samples and 497 LUAD samples. We observed a distinct difference in HSF2BP expression between lung tumor and nontumor samples $(\mathrm{P}=9.723 \mathrm{e}-27$; Figure 1). We then applied univariate analysis using data from 425 TCGA LUAD specimens (Figure 2A) and identified four risk factors: high HSF2BP expression, pathologic $\mathrm{N}$, pathologic $\mathrm{T}$, and tumor stage. The results of the multivariate Cox proportional hazard model found that HSF2BP and tumor stage were independent prognostic factors for progression of LUAD (Figure 2B).

\section{A significant impact of HSF2BP expression on prognosis of LUAD}

Aberrations in driver genes can result in a selective growth advantage for some cells, inducing tumor formation and further impairing prognosis in cancer patients. We used Kaplan-Meier survival analysis to explore the effect of HSF2BP on survival rates in 425 LUAD patients, including 212 high expression cases and 213 low expression cases $(\mathrm{P}=0.016$; Figure $2 C)$. The results indicated that HSF2BP posed a significant threat to survival status in LUAD, suggesting that HSF2BP was a critical gene in LUAD progression. In order to test the diagnostic value of HSF2BP, we generated receiver operating characteristic (ROC) curves of HSF2BP expression level in LUAD, calculating area under the curve (AUC) for 1, 3, and 5 years ( $\mathrm{AUC}=0.615,0.568$, and 0.583, respectively; Figure 2D). We found that HSF2BP played a mild diagnostic role in LUAD. Further exploration of the role of HSF2BP in the pathological mechanism and evolution of LUAD would be useful.

\section{An exploration between HSF2BP and methylation site changes in epigenetics}

DNA methylation is important for the growth and development of normal and abnormal cells. Alterations to the DNA methylation landscape could alter transcriptome levels and deregulate cellular pathways. To explore the impact of methylation conditions, we examined the methylation levels of six CpG sites of HSF2BP expression in 461 LUAD specimens from the University of California Santa Cruz (UCSC) database (Figure $3 A$ ) and then conducted Pearson correlation analysis between HSF2BP and the six methylation sites. We found a significant difference for cg05527272, which had a negative relationship with HSF2BP expression ( $\mathrm{R}=-0.14 ; \mathrm{P}=0.0084$; Figure $3 B$ ), revealing a role for methylation change in HSF2BP expression.

\section{A combination of HSF2BP expression and crucial signaling patbways to decode LUAD development by GSEA}

To explore the function of HSF2BP in LUAD, we utilized GSEA to identify known biological processes associated with our target gene (Figure 4). HSF2BP was demonstrated to impact a wide range of gene sets $[\mathrm{P}<0.05$; false discovery rate $(\mathrm{FDR})<0.25$; Table 1]. Of note, the cell cycle, DNA replication, nucleotide excision repair, mismatch repair, the P53 signaling pathway, ubiquitinmediated proteolysis, and homologous recombination were enriched in the HSF2BP high expression phenotype, providing a number of potential perspectives to fully explore the role of HSF2BP in LUAD evolution. Interestingly, we found that HSF2BP expression in LUAD was also significantly correlated with Alzheimer's disease.

\section{A latent interaction between HSF2BP expression and some oncogenes and tumor suppressor genes}

We employed coexpression analysis to process gene expression information of 551 lung tissue samples, identifying the top 5 genes positively and negatively related to HSF2BP expression, respectively (Table 2). We then generated a circular plot to visualize the coexpression results (Figure 5). 


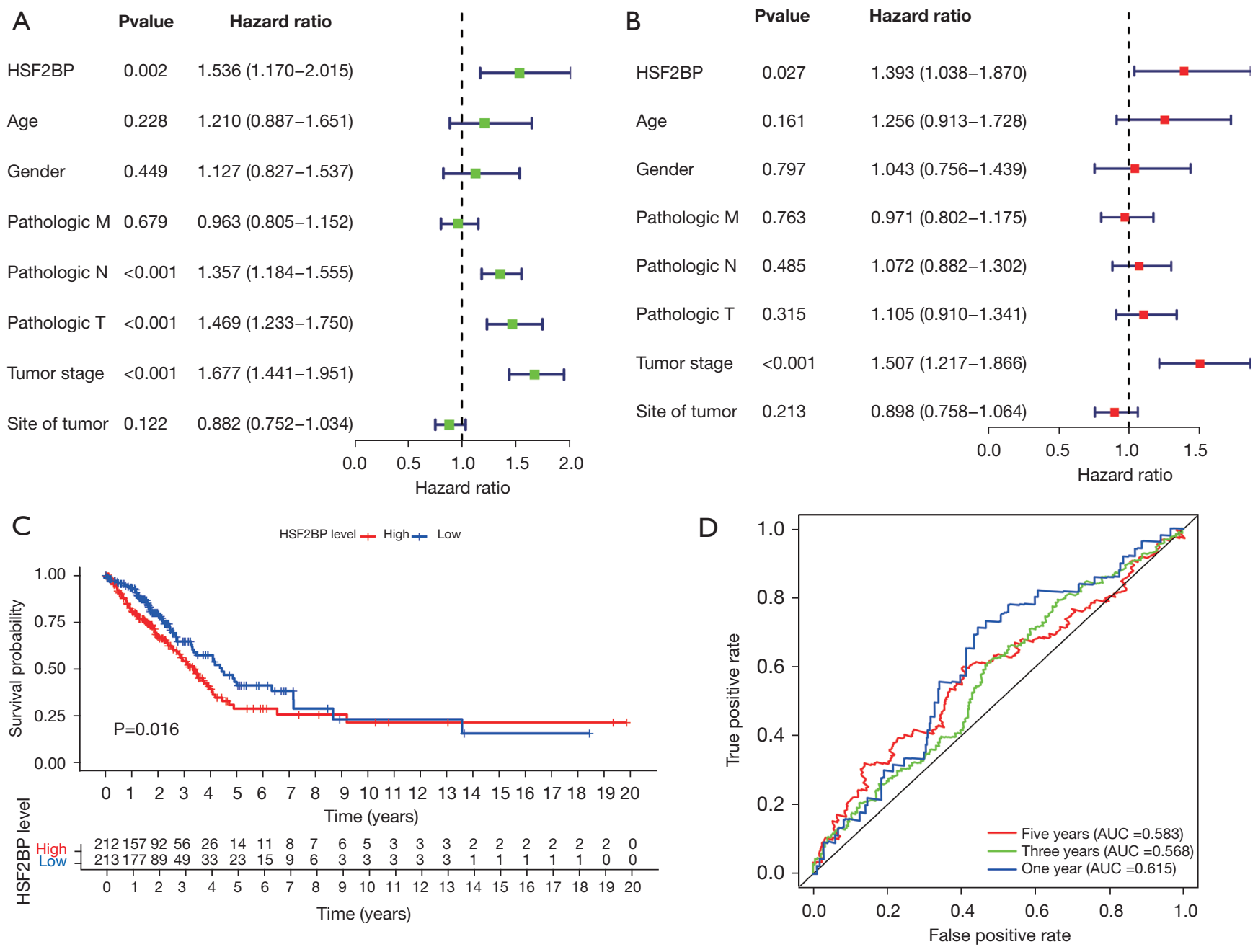

Figure 2 Bioinformatics analysis of HSF2BP using the TCGA database (LUAD; N=425). (A) Univariate analysis of HSF2BP; (B) multivariate analysis of HSF2BP; (C) survival analysis of patients with LUAD in the high HSF2BP and low HSF2BP groups. $\mathrm{P}=0.016$; (D) receiver operator characteristic curve analysis of HSF2BP. HSF2BP, heat shock transcription factor 2 binding protein; TCGA, The Cancer Genome Atlas; LUAD, lung adenocarcinoma; AUC, area under the curve.

HSF2BP expression was positively correlated with expression of MIS18A, PAIP1, CENPL, OIP5, and CDC7 and was negatively correlated with expression of TGFBR2, $C B X 7$, GNG7, HSD17B4, and DPYSL2. The genes included oncogenes (PAIP1, OIP5, and CDC7) and tumor suppressor genes (CBX7 and GNG7). In addition, TGFBR2 is regarded to be involved in tumor immunity.

\section{High HSF2BP expression is suspected to disturb tumor immunity}

We also made use of the TIMER database to further explore the role of HSF2BP in LUAD. Multivariable analysis showed that tumor stage was an independent prognostic risk factors in LUAD: stage 2 [HR $=2.541 ; 95 \%$ confidence interval (CI): $1.707-3.781 ; \mathrm{P}<0.001]$, stage 3 ( $\mathrm{HR}=3.072$; 95\% CI: 2.019-4.674; $\mathrm{P}<0.001)$, and stage $4(\mathrm{HR}=3.634$; 95\% CI: $2.011-6.565 ; \mathrm{P}<0.001) . \mathrm{CD}^{+} \mathrm{T}$ cells $(\mathrm{HR}=17.742$; 95\% CI: $1.267-248.494 ; \mathrm{P}=0.033)$ and high HSF2BP expression ( $\mathrm{HR}=1.330 ; 95 \% \mathrm{CI}: 1.037-1.705 ; \mathrm{P}=0.025$ ) were also independent prognostic risk factors in LUAD, while B cells (HR $=0.037$; 95\% CI: 0.003-0.540; $\mathrm{P}=0.016$ ) were a protective factor (Table 3). The relationships between HSF2BP expression and abundance of immune infiltrates showed that high expression of HSF2BP was negatively associated with $\mathrm{B}$ cells, $\mathrm{CD} 4^{+} \mathrm{T}$ cells, and dendritic cells 

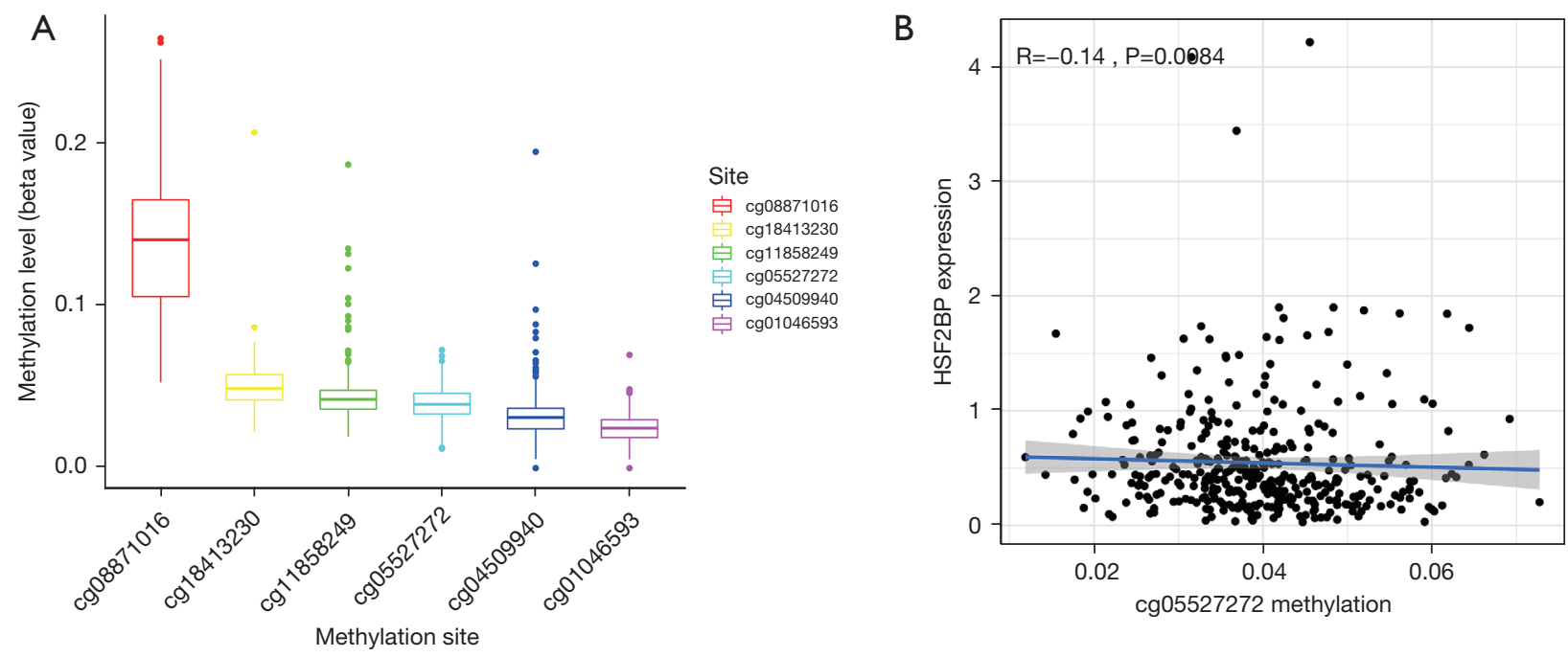

Figure 3 Regulation of HSF2BP by methylation. (A) Methylation level of 6 methylation sites of HSF2BP in LUAD; (B) correlation between cg05527272 methylation and HSF2BP expression. HSF2BP, heat shock transcription factor 2 binding protein; LUAD, lung adenocarcinoma.
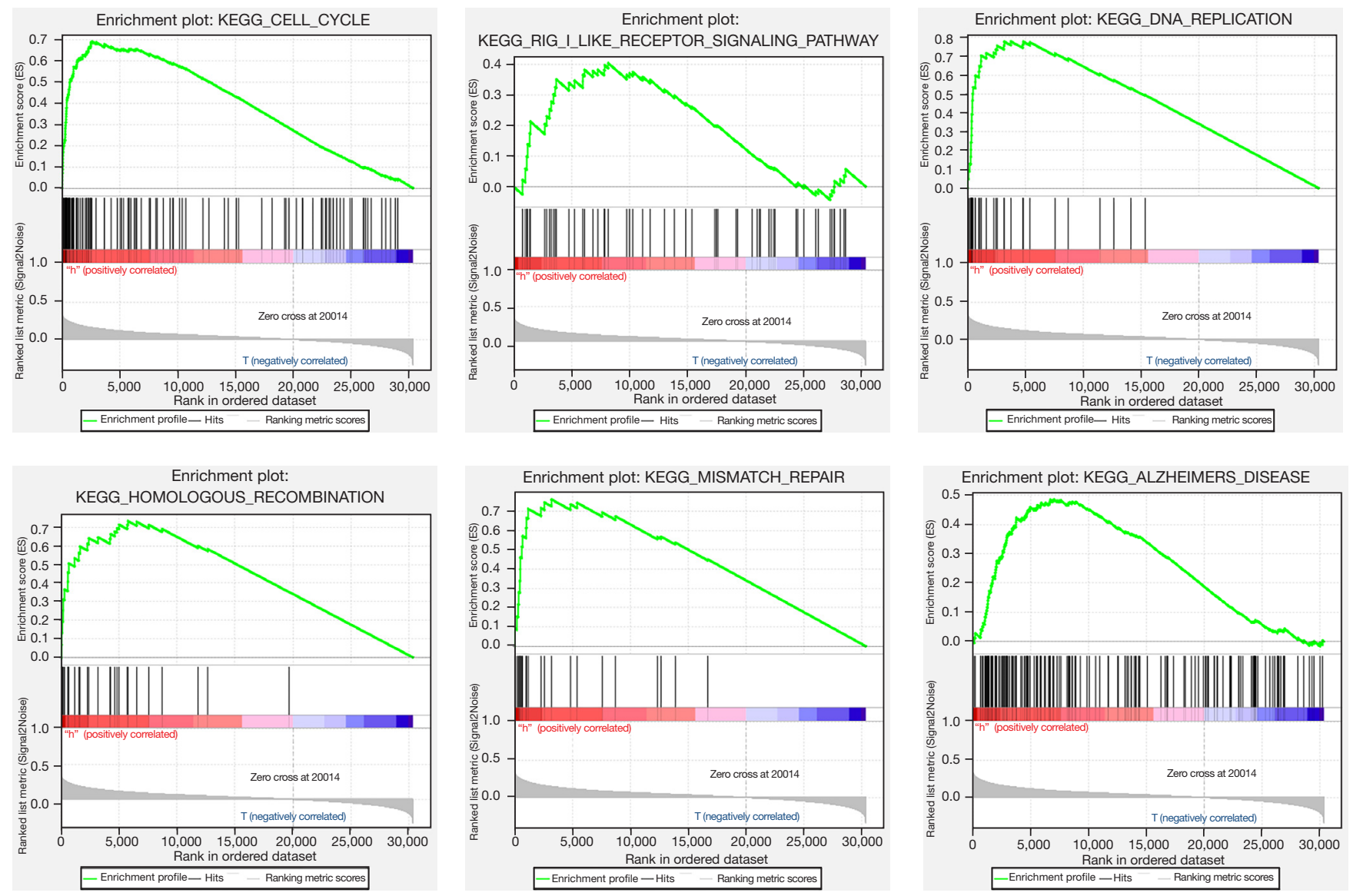

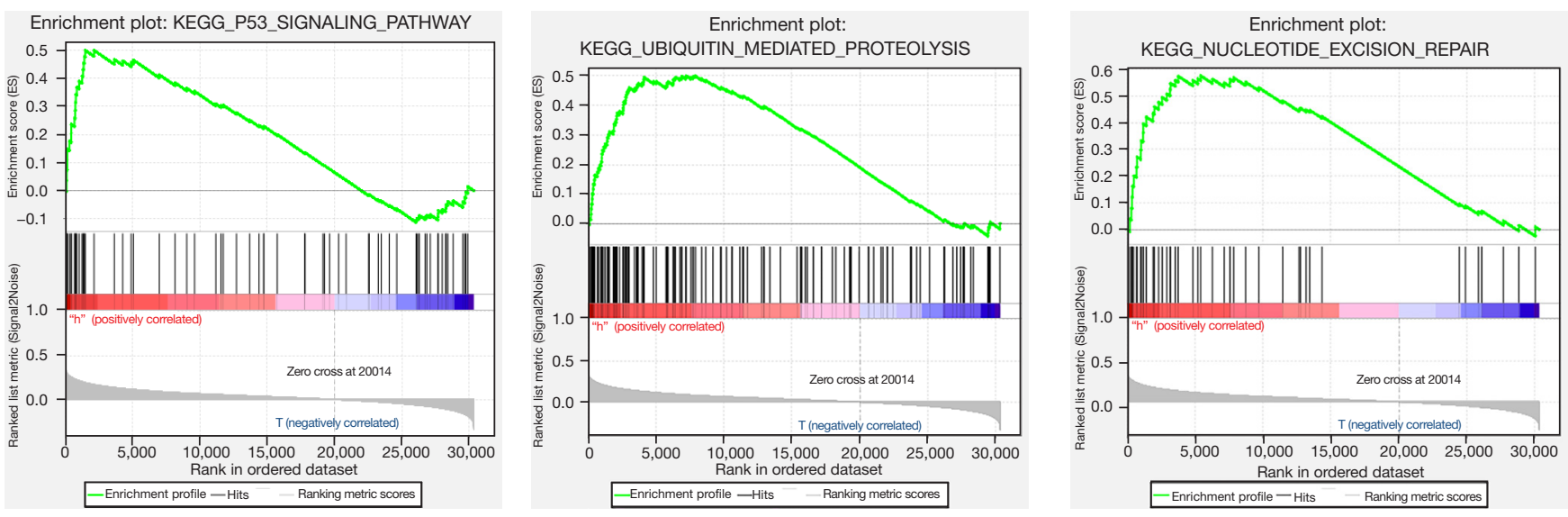

Figure 4 Nine significant cell signaling pathways enriched in high HSF2BP expression by GSEA analysis. HSF2BP, heat shock transcription factor 2 binding protein; GSEA, Gene Set Enrichment Analysis.

Table 1 Nine significant cell signaling pathways enriched in high HSF2BP expression

\begin{tabular}{lccc}
\hline Name & NES & P value & FDR \\
\hline Cell cycle & 2.426 & 0 & 0 \\
$\begin{array}{l}\text { Rig I like receptor signalin } \\
\text { g pathway }\end{array}$ & 1.582 & 0.037 & 0.119 \\
DNA replication & 1.932 & 0.004 & 0.017 \\
$\begin{array}{l}\text { Homologous } \\
\text { recombination }\end{array}$ & 2.063 & 0 & 0.012 \\
Mismatch repair & 2.005 & 0.002 & 0.013 \\
$\begin{array}{l}\text { Alzheimers disease } \\
\text { P53 signaling pathway }\end{array}$ & 1.702 & 0.027 & 0.082 \\
$\begin{array}{l}\text { Ubiquitin } \\
\text { mediated proteolysis }\end{array}$ & 2.055 & 0 & 0.011 \\
Nucleotide excision repair & 1.836 & 0.013 & 0.039 \\
\hline
\end{tabular}

HSF2BP, heat shock transcription factor 2 binding protein; NES, normalized enrichment score; FDR, false discovery rate

(Figure 6). Survival analysis revealed LUAD patients had better OS with increasing abundance of $B$ cells and dendritic cells, while high HSF2BP expression posed a threat to OS in LUAD patients (Figure 7). And we observed a significant negative correlation between HSF2BP and CD80 (Figure 8). Low expression of CD80 leads to tumor immune escape, as shown in LUAD patients through Gene Expression Profiling Interactive Analysis (GEPIA; http://gepia.cancer-pku.cn/; Figure 9). In addition, we also explored the correlation between SCNA of HSF2BP and
Table 2 Correlation between 10 known genes and HSF2BP expression (LUAD + normal, $\mathrm{N}=551$ )

\begin{tabular}{lcc}
\hline Gene & Cor & P value \\
\hline HSF2BP & 1 & 0 \\
MIS18A & 0.490 & $1.18 \mathrm{E}-34$ \\
PAIP1 & 0.481 & $2.65 \mathrm{E}-33$ \\
CENPL & 0.479 & $6.22 \mathrm{E}-33$ \\
OIP5 & 0.469 & $1.49 \mathrm{E}-31$ \\
CDC7 & 0.468 & $2.70 \mathrm{E}-31$ \\
TGFBR2 & -0.365 & $7.62 \mathrm{E}-19$ \\
CBX7 & -0.366 & $6.60 \mathrm{E}-19$ \\
GNG7 & -0.369 & $3.37 \mathrm{E}-19$ \\
HSD17B4 & -0.384 & $8.77 \mathrm{E}-21$ \\
DPYSL2 & -0.392 & $1.06 \mathrm{E}-21$
\end{tabular}

HSF2BP, heat shock transcription factor 2 binding protein; LUAD, lung adenocarcinoma; Cor, correlation.

abundance of immune infiltrates (Figure 10).

\section{Small-molecule drugs may have an effect in the treatment of LUAD}

Based on the outcomes of coexpression analysis, we explored the potential clinical treatment value of HSF2BP using the CMap database to identify four small-molecule drugs (Table 4): quinostatin (Figure 11A), MS-275 (Figure 11B), daunorubicin (Figure 11C), and menadione (Figure 11D). 


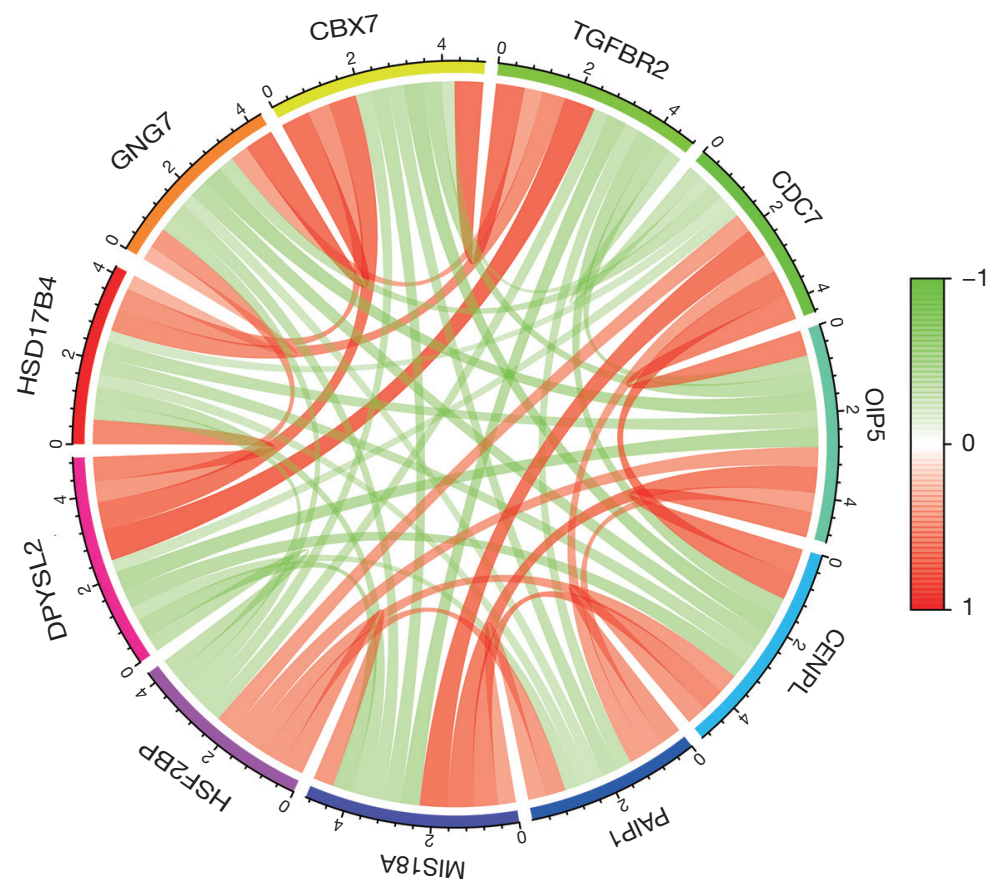

Figure 5 Circular plot of the top five genes positively and negatively correlated with the HSF2BP gene. Green represents negative association, and red represents positive association. HSF2BP, heat shock transcription factor 2 binding protein.

Table 3 Multivariable analysis of HSF2BP expression, clinical information and immune cells in LUAD $(\mathrm{N}=455)$

\begin{tabular}{lcccc}
\hline Variables & Coef & HR & $95 \%$ Cl & P value \\
\hline Stage 2 & 0.933 & 2.541 & $1.707-3.781$ & 0 \\
Stage 3 & 1.122 & 3.072 & $2.019-4.674$ & 0 \\
Stage 4 & 1.290 & 3.634 & $2.011-6.565$ & 0 \\
Purity & 0.200 & 1.221 & $0.556-2.681$ & 0.619 \\
B cell & -3.293 & 0.037 & $0.003-0.540$ & 0.016 \\
CD8 ${ }^{+}$T cell & 0.504 & 1.655 & $0.264-10.385$ & 0.591 \\
CD4 ${ }^{+}$T cell & 2.876 & 17.742 & $1.267-248.494$ & 0.033 \\
Macrophage & 0.397 & 1.487 & $0.096-22.992$ & 0.776 \\
Neutrophil & -1.855 & 0.156 & $0.003-7.010$ & 0.339 \\
Dendritic & -0.172 & 0.842 & $0.227-3.123$ & 0.797 \\
HSF2BP & 0.285 & 1.330 & $1.037-1.705$ & 0.025 \\
\hline
\end{tabular}

HSF2BP, heat shock transcription factor 2 binding protein; LUAD, lung adenocarcinoma; Coef, coefficient; HR, hazard ratio; $\mathrm{Cl}$, confidence interval.
In theory, these drugs could potentially improve the prognosis of LUAD.

\section{Meta-analysis of TCGA dataset and 3 GEO datasets of LUAD}

As no previous studies had identified HSF2BP expression as a risk factor in LUAD, we pooled the TCGA dataset (HR $=1.54 ; 95 \%$ CI: 1.17-2.02) with three GEO datasets [GSE31210 (HR =1.90; 95\% CI: 1.27-2.83), GSE37745 (HR =1.09; 95\% CI: 0.73-1.62), and GSE50081 (HR =1.81; 95\% CI: 1.13-2.89)] to perform a meta-analysis to confirm the role of HSF2BP in LUAD. The pooled HR and 95\% CI for the association between high HSF2BP expression and OS in 958 cases of LUAD patients was 1.53 (1.28-1.83), and no significant heterogeneity was observed among the four datasets $\left(\mathrm{I}^{2}=32 \%<50 \% ; \mathrm{P}=0.22\right.$; Figure 12). Overall, high expression of HSF2BP was considered an independent prognostic risk factor for patients with LUAD. 

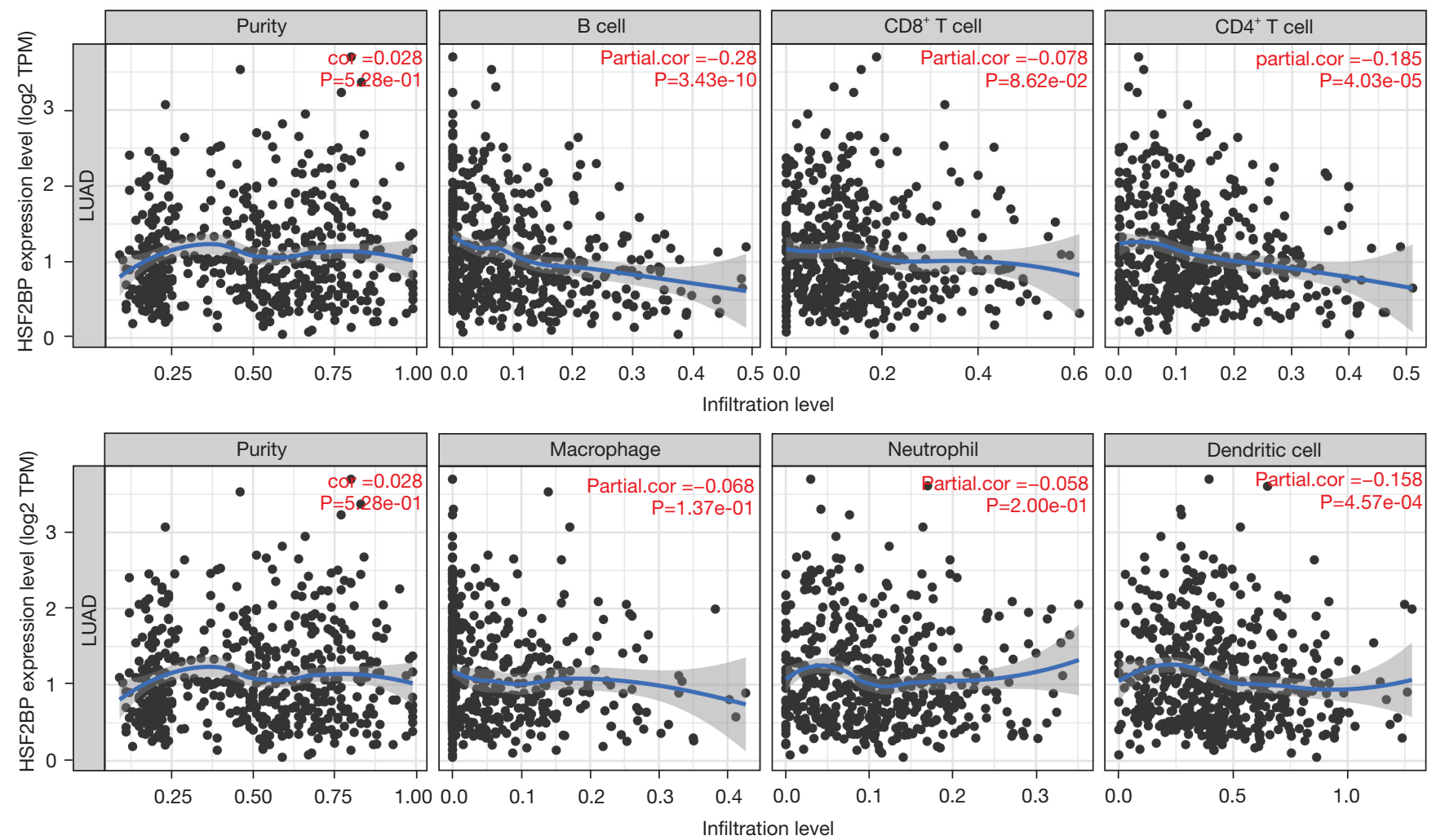

Figure 6 Correlation between the expression of HSF2BP and immune cell infiltration. HSF2BP, heat shock transcription factor 2 binding protein; LUAD, lung adenocarcinoma.

\section{Discussion}

LUAD is a common cancer with high mortality (1). Due to the poor prognosis in LUAD patients, particularly for advanced and metastatic stages, conventional treatments such as surgical resection, chemotherapy, and radiotherapy are relatively ineffective for curbing the progress of LUAD (18). In recent years, molecular-targeted therapies involving EGFR, ALK, ROS1, BRAFV600E, MET, and RET and immunotherapy that targets programmed cell death 1 (PD-1) and its ligand (PD-L1) have been employed in the management of LUAD, making a remarkable difference (19). However, due to tumor heterogeneity and drug resistance, novel therapeutic methods are urgently needed. Encouragingly, the role of HSF2BP in tumorigenesis is gradually being revealed, providing a potentially targetable vulnerability $(14,15,19)$. Currently, the status of HSF2BP in LUAD remains unclear and the impact of HSF2BP on the pathogenesis of LUAD requires further, systematic research.

In our study, we first made use of the TCGA and UCSC databases to explore the clinical features and prognostic value of HSF2BP expression for LUAD based on transcriptome data and HSF2BP methylation. LUAD samples had a higher expression of HSF2BP than normal samples, indirectly indicating that it was likely involved in the progress of LUAD. Further support for our theory was provided by the finding that overexpression of HSF2BP led to poorer OS. Multivariable Cox analysis verified that elevated HSF2BP expression acted as a significant and independent risk factor in the evolution of LUAD, along with tumor stage. However, the diagnostic value of our target gene was relatively moderate. To overcome the limitation of using a single database source, a metaanalysis including 958 LUAD specimens obtained from one TCGA and three GEO datasets was carried out, providing strong evidence that high expression of HSF2BP was an independent prognostic factor in LUAD. As a key cause of epigenetic alterations in cancer, DNA methylation aberrations result in chromosomal instability and abnormal gene expression through dysregulation of methylation levels at $\mathrm{CpG}$ islands (20). Increasing evidence has shown that aberrant DNA methylation plays a crucial 

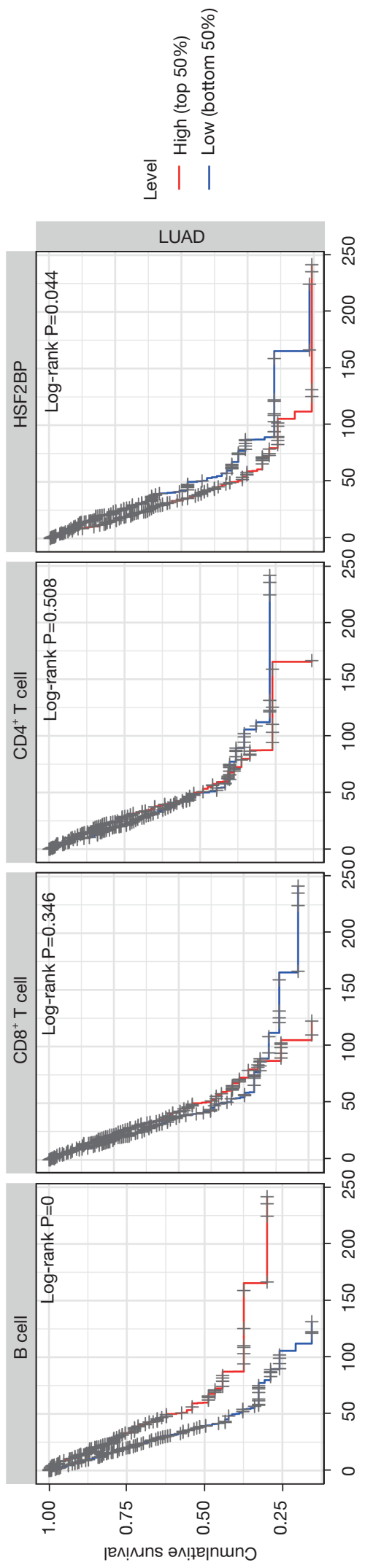
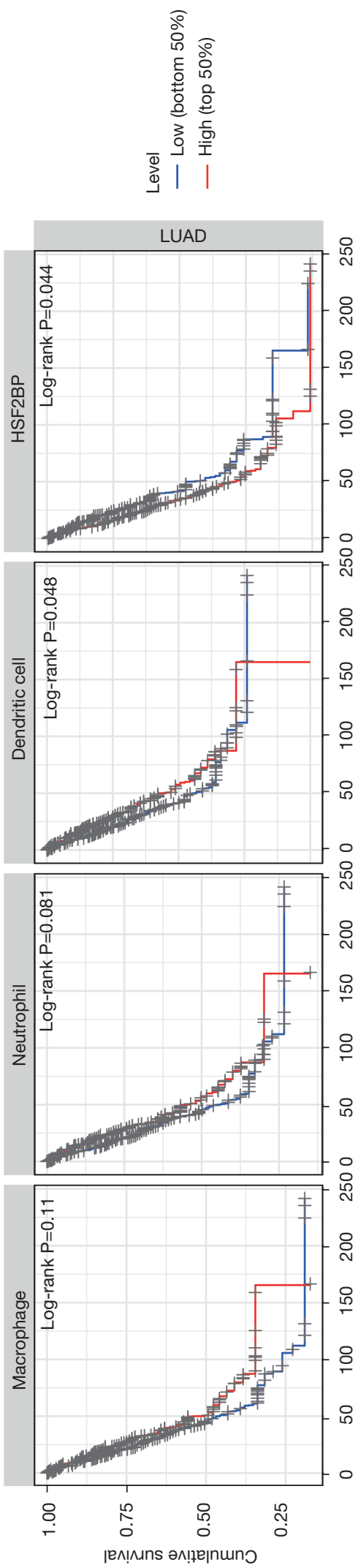

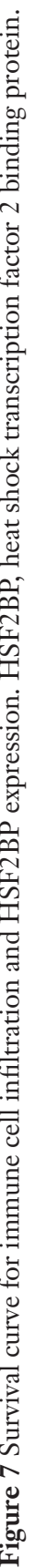




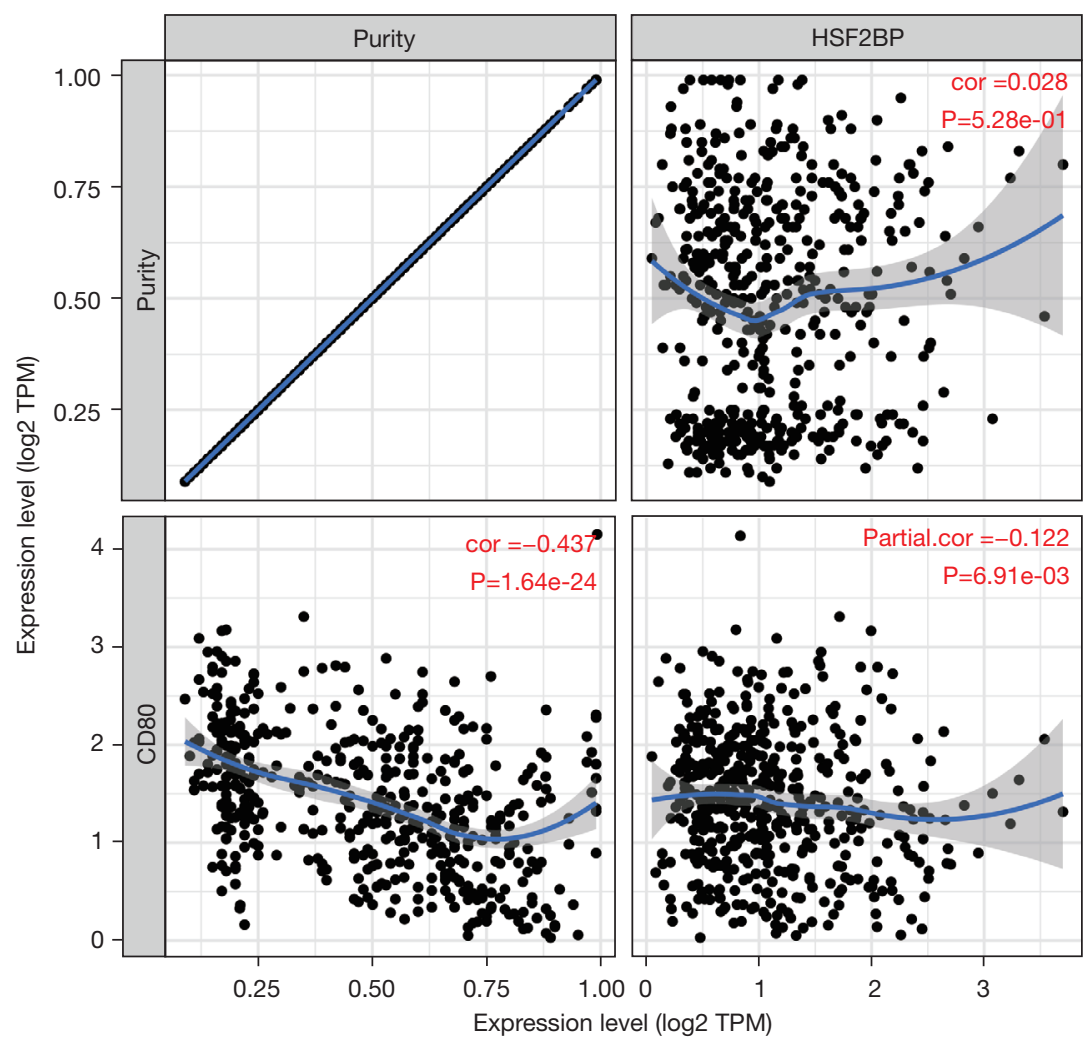

Figure 8 Relationship between CD80 and HSF2BP expression in LUAD (N=515). HSF2BP, heat shock transcription factor 2 binding protein; LUAD, lung adenocarcinoma; TPM, transcripts per million.

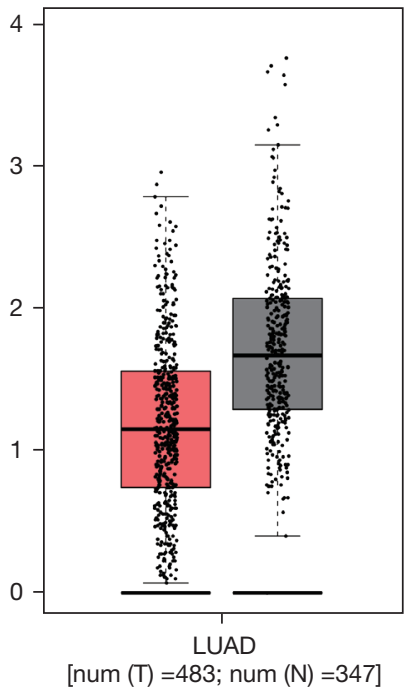

Figure 9 CD80 expression in LUAD (N=483) and normal $(\mathrm{N}=347)$ tissues of human lung. LUAD, lung adenocarcinoma. role in the malignant transformation and progression of LUAD $(20,21)$. We investigated whether HSF2BP methylation status affected the expression of HSF2BP in LUAD. We found that of six methylation sites, one had a significant correlation with HSF2BP expression. However, the relationship between global methylation level and expression of HSF2BP was not evident in LUAD, suggesting that the diverse locations of gene methylation exerted varying influence on gene expression (22). We found that the methylation level of cg05527272 negatively regulated the expression of HSF2BP, which suggested that cg05527272 hypomethylation might have led to high HSF2BP expression.

A growing number of cell signaling pathways have been shown to be involved in tumorigenesis and are being targeted to halt the progression of cancer (23). We observed through GSEA analysis that high expression of HSF2BP 


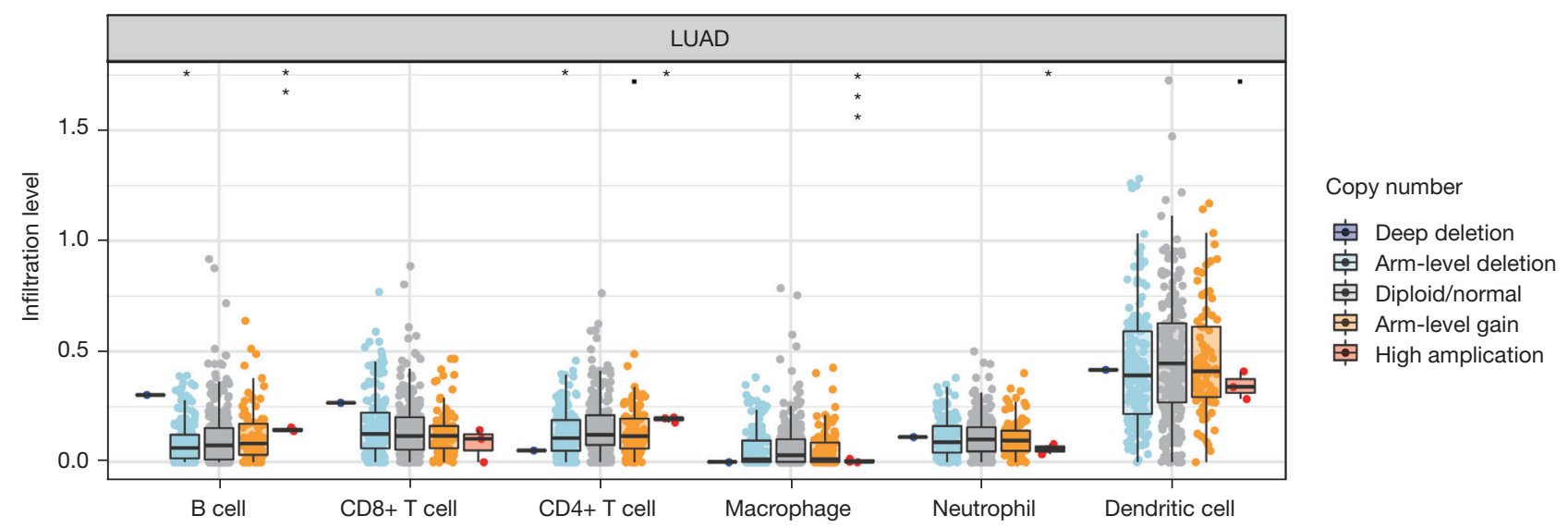

Figure 10 Relevance between copy number alterations of HSF2BP and immune infiltration level in different immune cells in LUAD. *, $\mathrm{P}<0.05$; **, $\mathrm{P}<0.005$; **, $\mathrm{P}<0.001$. HSF2BP, heat shock transcription factor 2 binding protein; LUAD, lung adenocarcinoma.

Table 4 Four potential small molecular drugs in LUAD

\begin{tabular}{lccc}
\hline Name & Mean & Enrichment & P value \\
\hline Quinostatin & -0.916 & -0.977 & 0.001 \\
MS-275 & -0.763 & -0.919 & 0.013 \\
Menadione & -0.703 & -0.843 & 0.050 \\
Daunorubicin & -0.699 & -0.816 & 0.002 \\
\hline
\end{tabular}

LUAD, lung adenocarcinoma. was associated with a number of cell signaling pathways, including the cell cycle, mismatch repair, homologous recombination, P53 signaling pathway, ubiquitin mediatedproteolysis, and some metabolism pathways. Further, the cell cycle, mismatch repair, homologous recombination, P53 signaling pathway, and ubiquitin-mediated proteolysis are typical signaling pathways for maintaining genomic stability and a normal cell cycle, and dysregulation of these
A<smiles>CCc1ccc(C(=O)c2cnc3cc4c(cc3c2N2CCC(C(N)=O)CC2)OCCO4)cc1</smiles>

B<smiles>Nc1ccccc1NC(=O)c1ccc(CNC(=O)OCc2cccnc2)cc1</smiles>

C<smiles>COc1cccc2c1C(=O)c1c(O)c3c(c(O)c1C2=O)C[C@@](O)(C(C)=O)C[C@@H]3O[C@H]1C[C@H](N)[C@@H](O)[C@H](C)O1</smiles>

D<smiles>CC1=CC(=O)c2ccccc2C1=O</smiles>

Figure 11 A potential treatment of four small molecular drugs in LUAD. (A) Quinostatin. CID, 6620134. $\mathrm{C}_{26} \mathrm{H}_{27} \mathrm{~N}_{3} \mathrm{O}_{4}$; (B) MS-275. CID, 4261. $\mathrm{C}_{21} \mathrm{H}_{20} \mathrm{~N}_{4} \mathrm{O}_{3}$; (C) daunorubicin. CID, 30323. $\mathrm{C}_{27} \mathrm{H}_{29} \mathrm{NO}_{10}$; (D) menadione. CID, 4055. $\mathrm{C}_{11} \mathrm{H}_{8} \mathrm{O}_{2}$. LUAD, lung adenocarcinoma; CID, compound identifier. 


$\begin{array}{lcc}\text { Study } & \text { TE } & \text { seTE } \\ \text { GSE31210 } & 0.64 & 0.2035 \\ \text { GSE37745 } & 0.08 & 0.2043 \\ \text { GSE50081 } & 0.59 & 0.2401 \\ \text { TCGA RNA-Seq } & 0.43 & 0.1386 \\ \text { Fixed effect model } & & \\ \text { Heterogeneity: } I^{2}=32 \%, \tau^{2}=0.0173, P=0.22\end{array}$

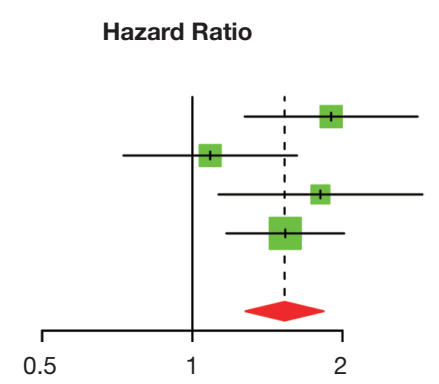

$\begin{array}{ccc}\text { HR } & \mathbf{9 5 \%} \text { Cl } & \text { Weight } \\ & & \\ 1.90 & {[1.27 ; 2.83]} & 20.6 \% \\ 1.09 & {[0.73 ; 1.62]} & 20.4 \% \\ 1.81 & {[1.13 ; 2.89]} & 14.8 \% \\ 1.54 & {[1.17 ; 2.02]} & 44.3 \% \\ & & \\ 1.53 & {[1.28 ; 1.83]} & 100.0 \%\end{array}$

Figure 12 Forest plot of high HSF2BP expression in LUAD from four datasets. HSF2BP, heat shock transcription factor 2 binding protein; LUAD, lung adenocarcinoma; TE, estimate of treatment effect; se'TE, standard error of treatment estimate; HR, hazard ratio; CI, confidence interval.

would give rise to malignant mutations and unlimited proliferation in tumorigenesis (24-26). The DNA mismatch repair pathway is crucial in maintaining genomic stability, loss of which can induce tumorigenesis (24). Deregulation of the cell cycle would result in aberrant cell proliferation, characterizing cancer (26). In addition, HSF2BP enables homologous recombination to be held back in DNA interstrand crosslink repair, and the breast cancer gene (BRCA)2-HSF2BP-BRME1 complex can control meiotic recombination and impede BRCA2-RAD51 function in cancer cells $(14,17)$. HSF2BP is likely to be involved in these signaling pathways, thereby increasing genomic instability and contributing to malignant progression of LUAD.

The activation of oncogenes and inactivation of tumor suppressor genes participate in and promote the development of cancer (27). In our study, the 10 genes most associated with HSF2BP expression, including the top 5 positive and negative associations, were identified through coexpression to further explore the role of HSF2BP in LUAD. Of note, PAIP1, OIP 5, and CDC7 have been widely reported to act as oncogenes in diverse solid malignant tumors, including cervical cancer, pancreatic cancer, breast cancer, liver cancer, bladder cancer, and LUAD (28-30). PAIP1 depletion by RNA interference could significantly inhibit cell proliferation and lead to cell cycle arrest in breast cancer (28). Elevated OIP5 expression, via BMPR2/JUN/CHEK1/RAC1 dysregulation, promotes the progression of liver cancer (29). Conversely, it has been reported that CBX7 and GNG7 play a tumor suppressor role in the development of some cancers, and TGFBR2 has been reported to regulate the transcription of genes related to immunosuppression and tumorigenesis (31-34). It is worth noting that the subtle interplay between CBX7 and
E-box could undermine TWIST1 function, tumorigenicity, and metastatic tendency (31). Moreover, recovering CBX7 expression leads to human lung carcinoma cells becoming susceptible to irinotecan treatment (32). In short, HSF2BP participates in some cell signaling pathways and interacts with other driver genes, resulting in the evolution of LUAD.

Tumor immune escape enables tumor cells to avoid recognition and attack by the immune system through multiple mechanisms, resulting in proliferation and metastasis. This process has become a focus of cancer research recently, particularly the role of PD-1 and PDL1 (35). Previous research has mainly focused on T cells, although some studies have demonstrated that other immune cells may also be effective in promoting an antitumor immune response $(36,37)$. A recent study in Nature once again emphasized that B cells and tertiary lymphoid structures promoted antitumor immunity and immunotherapy response (38). Our results showed that the infiltration level of B cells, which was negatively associated with HSF2BP expression, was an independent prognostic factor and contributed to improve OS in LUAD patients, consistent with the findings of the research mentioned above. Further, we found that HSF2BP expression was also negatively related to dendritic cells and CD80, which is located on the surface of antigen presenting cells (APC) and able to bind with CD28 on T cells and thereby promotes $\mathrm{T}$ cell response $(39,40)$. However, low CD80 expression in APC intensifies tumor immune escape in LUAD, suggesting that high HSF2BP expression may undermine $\mathrm{T}$ cell response and lead to immune escape through mechanisms that potentially downregulate CD80 expression. Immunotherapy for LUAD has achieved success through tumor immune escape blockage and improvement of 
systemic antitumor responses of $T$ cells, particularly the use of anti-PD-L1 and anti-PD-1 therapy (41). Despite these positive results, immunotherapy continues to be ineffective for many LUAD patients. Increasingly, studies have been reporting that some small-molecule drugs may have synergy with immunotherapy by modifying molecular pathways to remove immune suppression and tolerance $(41,42)$. Combining coexpression analysis and the CMap database, we identified four small-molecule drugs which might facilitate the treatment of LUAD, including quinostatin, MS-275, daunorubicin, and menadione. Clinical trials have explored the use of MS-275, a histone deacetylase inhibitor (HDACi), to reverse drug resistance and enhance clinical efficacy (43), while menadione (vitamin k3) is widely used in the treatment of coagulation disorders. Recent studies have reported that menadione reduced the risk of cancer through antiproliferative, autophagic, and proapoptotic effects $(44,45)$. The use of small-molecule drugs to enhance and improve the efficacy of LUAD treatment in the future appears promising.

Our research had some limitations. First, the TCGA database contains far more tumor tissue samples than normal samples, which may have influenced our results. However, a meta-analysis incorporating three GEO datasets in addition to the TCGA RNA-seq data provided further support for our findings. Second, we could not validate the role of HSF2BP methylation in LUAD patients due to the lack of methylation information on the GEO database. Third, the expression of HSF2BP in LUAD will require additional verification, and our future research will focus on filling these gaps with clinical experimental data. Finally, although our comprehensive investigation revealed, for the first time, the role of HSF2BP in LUAD, more research is required to further explore the function of HSF2 BP expression in LUAD.

\section{Conclusions}

Overexpression of HSF2BP indicated a worse prognosis in LUAD patients. HSF2BP expression potentially contributed to tumor immune escape through the regulation of B cells, dendritic cells, and CD80 and to genomic instability by impeding homologous recombination, mismatch repair, and the cell cycle, thereby promoting development and progression of LUAD. These findings reveal that HSF2BP may have potential as a novel prognostic and therapeutic target. Simultaneously, small-molecule drugs identified through our research may lead to improvements in the treatment of advanced LUAD in the future.

\section{Acknowledgments}

We gratefully acknowledge contributions from GEO and TCGA Research Network. The first author particularly appreciated the support from Department of Thoracic Surgery, Zhengzhou University People's Hospital, Henan Provincial People's Hospital.

Funding: "23456 Talent Project" of Henan Provincial People's Hospital \& Medical Service Capacity Improvement Project in Henan Provincial Medical Institutions.

\section{Footnote}

Reporting Checklist: The authors have completed the STARD reporting checklist. Available at https://dx.doi. org/10.21037/atm-21-4935

Conflicts of Interest: All authors have completed the ICMJE uniform disclosure form (available at https://dx.doi. org/10.21037/atm-21-4935). The authors have no conflicts of interest to declare.

Ethical Statement: The authors are accountable for all aspects of the work in ensuring that questions related to the accuracy or integrity of any part of the work are appropriately investigated and resolved. The study was conducted in accordance with the Declaration of Helsinki (as revised in 2013).

Open Access Statement: This is an Open Access article distributed in accordance with the Creative Commons Attribution-NonCommercial-NoDerivs 4.0 International License (CC BY-NC-ND 4.0), which permits the noncommercial replication and distribution of the article with the strict proviso that no changes or edits are made and the original work is properly cited (including links to both the formal publication through the relevant DOI and the license). See: https://creativecommons.org/licenses/by-nc-nd/4.0/.

\section{References}

1. Chen J, Yang H, Teo ASM, et al. Genomic landscape of lung adenocarcinoma in East Asians. Nat Genet 2020;52:177-86.

2. National Lung Screening Trial Research Team; Church TR, Black WC, et al. Results of initial low-dose computed 
tomographic screening for lung cancer. N Engl J Med 2013;368:1980-91.

3. Yang CY, Yang JC, Yang PC. Precision management of advanced non-small cell lung cancer. Annu Rev Med 2020;71:117-36.

4. Yang WC, Hsu FM, Yang PC. Precision radiotherapy for non-small cell lung cancer. J Biomed Sci 2020;27:82.

5. Zhang C, Leighl NB, Wu YL, et al. Emerging therapies for non-small cell lung cancer. J Hematol Oncol 2019; $12: 45$.

6. Quintanal-Villalonga Á, Chan JM, Yu HA, et al. Lineage plasticity in cancer: a shared pathway of therapeutic resistance. Nat Rev Clin Oncol 2020;17:360-71.

7. Wu J, Liu T, Rios Z, et al. Heat shock proteins and cancer. Trends Pharmacol Sci 2017;38:226-56.

8. Saini J, Sharma PK. Clinical, prognostic and therapeutic significance of heat shock proteins in cancer. Curr Drug Targets 2018;19:1478-90.

9. Yoshima T, Yura T, Yanagi H. Novel testis-specific protein that interacts with heat shock factor 2. Gene 1998;214:139-46.

10. Puustinen MC, Sistonen L. Molecular mechanisms of heat shock factors in cancer. Cells 2020;9:1202.

11. Ciocca DR, Calderwood SK. Heat shock proteins in cancer: diagnostic, prognostic, predictive, and treatment implications. Cell Stress Chaperones 2005;10:86-103.

12. Ritossa FMJE. A new puffing pattern induced by temperature shock and DNP in drosophila. Cell Mol Life Sci 1962;18:571-3.

13. Zhong YH, Cheng HZ, Peng H, et al. Heat shock factor 2 is associated with the occurrence of lung cancer by enhancing the expression of heat shock proteins. Oncol Lett 2016;12:5106-12.

14. Zhang J, Gurusaran M, Fujiwara Y, et al. The BRCA2MEILB2-BRME1 complex governs meiotic recombination and impairs the mitotic BRCA2-RAD51 function in cancer cells. Nat Commun 2020;11:2055.

15. Brandsma I, Sato K, van Rossum-Fikkert SE, et al. HSF2BP interacts with a conserved domain of BRCA2 and is required for mouse spermatogenesis. Cell Rep 2019;27:3790-3798.e7.

16. Tang Z, Li C, Kang B, et al. GEPIA: a web server for cancer and normal gene expression profiling and interactive analyses. Nucleic Acids Res 2017;45:W98-W102.

17. Sato K, Brandsma I, van Rossum-Fikkert SE, et al. HSF2BP negatively regulates homologous recombination in DNA interstrand crosslink repair. Nucleic Acids Res 2020;48:2442-56.
18. Denisenko TV, Budkevich IN, Zhivotovsky B. Cell deathbased treatment of lung adenocarcinoma. Cell Death Dis 2018;9:117.

19. Ettinger DS, Wood DE, Aggarwal C, et al. NCCN guidelines insights: non-small cell lung cancer, version 1.2020. J Natl Compr Canc Netw 2019;17:1464-72.

20. Kerr KM, Galler JS, Hagen JA, et al. The role of DNA methylation in the development and progression of lung adenocarcinoma. Dis Markers 2007;23:5-30.

21. Lou-Qian Z, Rong Y, Ming L, et al. The prognostic value of epigenetic silencing of p16 gene in NSCLC patients: a systematic review and meta-analysis. PLoS One 2013;8:e54970.

22. Koch A, Joosten SC, Feng Z, et al. Analysis of DNA methylation in cancer: location revisited. Nat Rev Clin Oncol 2018;15:459-66.

23. Clara JA, Monge C, Yang Y, et al. Targeting signalling pathways and the immune microenvironment of cancer stem cells - a clinical update. Nat Rev Clin Oncol 2020;17:204-32.

24. Gupta D, Heinen CD. The mismatch repair-dependent DNA damage response: Mechanisms and implications. DNA Repair (Amst) 2019;78:60-9.

25. Muller PA, Vousden KH. Mutant p53 in cancer: new functions and therapeutic opportunities. Cancer Cell 2014;25:304-17.

26. Williams GH, Stoeber K. The cell cycle and cancer. J Pathol 2012;226:352-64.

27. Kontomanolis EN, Koutras A, Syllaios A, et al. Role of oncogenes and tumor-suppressor genes in carcinogenesis: a review. Anticancer Res 2020;40:6009-15.

28. Piao J, Chen L, Jin T, et al. Paip1 affects breast cancer cell growth and represents a novel prognostic biomarker. Hum Pathol 2018;73:33-40.

29. Li Y, Xiao F, Li W, et al. Overexpression of Opa interacting protein 5 increases the progression of liver cancer via BMPR2/JUN/CHEK1/RAC1 dysregulation. Oncol Rep 2019;41:2075-88.

30. Cao JX. miR-888 regulates cancer progression by targeting multiple targets in lung adenocarcinoma. Oncol Rep 2019;41:3367-76.

31. Li J, Alvero AB, Nuti S, et al. CBX7 binds the E-box to inhibit TWIST-1 function and inhibit tumorigenicity and metastatic potential. Oncogene 2020;39:3965-79.

32. Cacciola NA, Sepe R, Forzati F, et al. Restoration of CBX7 expression increases the susceptibility of human lung carcinoma cells to irinotecan treatment. Naunyn Schmiedebergs Arch Pharmacol 2015;388:1179-86. 
33. Xu S, Zhang H, Liu T, et al. G Protein $\gamma$ subunit 7 loss contributes to progression of clear cell renal cell carcinoma. J Cell Physiol 2019;234:20002-12.

34. Akhurst RJ. Targeting TGF- $\beta$ signaling for therapeutic gain. Cold Spring Harb Perspect Biol 2017;9:a022301.

35. Jiang X, Wang J, Deng X, et al. Role of the tumor microenvironment in PD-L1/PD-1-mediated tumor immune escape. Mol Cancer 2019;18:10.

36. Sarvaria A, Madrigal JA, Saudemont A. B cell regulation in cancer and anti-tumor immunity. Cell Mol Immunol 2017;14:662-74.

37. Tsou P, Katayama H, Ostrin EJ, et al. The emerging role of B cells in tumor immunity. Cancer Res 2016;76:5597-601.

38. Helmink BA, Reddy SM, Gao J, et al. B cells and tertiary lymphoid structures promote immunotherapy response. Nature 2020;577:549-55.

39. Chen R, Ganesan A, Okoye I, et al. Targeting B7-1 in immunotherapy. Med Res Rev 2020;40:654-82.

Cite this article as: Huang Z, Liu Z, Cheng X, Han Z, Li J, Xia T, Gao Y, Wei L. Prognostic significance of HSF2BP in lung adenocarcinoma. Ann Transl Med 2021;9(20):1559. doi: 10.21037/atm-21-4935
40. Greenwald RJ, Freeman GJ, Sharpe AH. The B7 family revisited. Annu Rev Immunol 2005;23:515-48.

41. Han C, Zhang A, Liu Z, et al. Small molecular drugs reshape tumor microenvironment to synergize with immunotherapy. Oncogene 2021;40:885-98.

42. van der Zanden SY, Luimstra JJ, Neefjes J, et al. Opportunities for small molecules in cancer immunotherapy. Trends Immunol 2020;41:493-511.

43. Mamdani H, Jalal SI. Histone deacetylase inhibition in non-small cell lung cancer: hype or hope? Front Cell Dev Biol 2020;8:582370.

44. Dahlberg S, Ede J, Schött U. Vitamin K and cancer. Scand J Clin Lab Invest 2017;77:555-67.

45. Ivanova D, Zhelev Z, Getsov P, et al. Vitamin K: Redoxmodulation, prevention of mitochondrial dysfunction and anticancer effect. Redox Biol 2018;16:352-8.

(English Language Editor: A. Muijlwijk) 